\title{
Kein Vorteil für Ticagrelor bei PAVK
}

\author{
Bei Patienten mit akutem Koronarsyndrom ist Ticagrelor dem alten Standard Clopidogrel überlegen. \\ Für die symptomatische periphere arterielle Verschlusskrankheit (PAVK) gilt dies jedoch nicht.
}

\begin{abstract}
Patienten mit symptomatischer PAVK haben ein deutlich erhöhtes Risiko für kardiovaskuläre Ereignisse. Für die Sekundärprävention bei PAVK-Patienten mit stabiler kardiovaskulärer Erkrankung wird Clopidogrel statt Acetylsalicylsäure empfohlen. Allerdings zeigte sich in jüngeren Studien Ticagrelor gegenüber Clopidogrel als überlegen hinsichtlich kardiovaskulärer Ereignisse und Mortalität. Dies gilt für Patienten mit akutem Koronarsyndrom sowie nach zurückliegendem Myokardinfarkt - und insbesondere auch für Patienten mit begleitender PAVK.

Ziel der großen, randomisierten, doppelblinden EUCLID-Studie war es daher,
\end{abstract}

Ticagrelor $(2 \times 90 \mathrm{mg} / \mathrm{d})$ gegen Clopidogrel $(1 \times 75 \mathrm{mg} / \mathrm{d})$ an 13.885 Patienten mit PAVK zu vergleichen. Der primäre Endpunkt kombinierte kardiovaskulären Tod, Myokardinfarkt und Schlaganfall. Die mittlere Nachbeobachtungsdauer betrug 30 Monate.

Im Ergebnis zeigte sich im primären Endpunkt kein signifikanter Unterschied zwischen den Behandlungsgruppen (Hazard Ratio 1,02, p = 0,65). Auch die Risiken für kritische Extremitätenischämien sowie für schwere Blutungen unterschieden sich nicht signifikant.

- Hiatt WR, Fowkes FG, Heizer G et al. Ticagrelor versus clopidogrel in symptomatic peripheral artery disease. $N$ Engl J Med. 2017;376:32-40

\section{KOMMENTAR}

Die EUCLID-Studie ist im Ausgang negativ: Ticagrelor als Monotherapie bietet keinen Vorteil gegenüber Clopidogrel bei PAVK. Es zeigte sich jedoch, dass sich das kardiovaskuläre Risikopotenzial und die antithrombozytären Behandlungergebnisse bei Patienten mit klinisch führender PAVK (und z. T. begleitender $K H K$ ) von jenen mit führender KHK (und z. T. begleitender PAVK) deutlich unterscheiden können. Dies muss noch genauer untersucht werden, gerade weil die Raten kardiovaskulärer Ereignisse bei Patienten mit signifikanter PAVK weiterhin hoch sind.

Prof. Dr. med. P. W. Radke

\section{Jeder vierte Hochbetagte in Deutschland ist Typ-2-Diabetiker}

Es klingt erstaunlich, aber man weiß eigentlich gar nicht genau, wie hoch die Prävalenz von Diabetes mellitus in Deutschland ist. Auf kleineren Kohortenstudien beruhende Schätzungen schwanken zwischen $6 \%$ und $12 \%$. Düsseldorfer Forscher hatten nun die Möglichkeit, die Datensätze von rund 65 Millionen Menschen auszuwerten, die 2009 und 2010 in der GKV versichert waren. Gefiltert wurde nach ICD-kodierten Diabetesdiagnosen aus dem ambulanten und dem stationären Sektor.

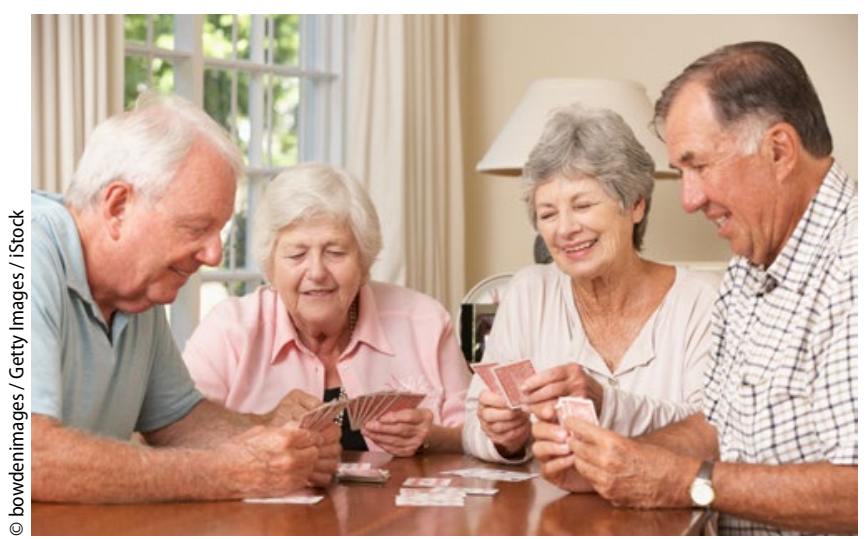

Einer von ihnen leidet wohl an Typ-2-Diabetes.
Demnach hatten im Jahr 2009 etwa 6,4 von 65,6 Millionen GKVVersicherten Diabetes. 2010 waren es 6,7 von 64,9 Millionen. Das entspricht einer Prävalenz von 9,7\% bzw. 9,9\%. Vom Typ-2-Diabetes waren 4,6 bzw. 4,7 Millionen Versicherte betroffen. Hochgerechnet auf die Gesamtbevölkerung hieße das, dass in diesem Zeitraum 5,8 Millionen Typ-2-Diabetiker in Deutschland lebten.

Die Ergebnisse liegen eher am oberen Rand dessen, was diskutiert wurde. Gerade in Bezug auf ältere Patienten bietet die Studie erstmalig verlässliche Aussagen: Offenbar ist ein Viertel der Menschen über 80 in Deutschland an Typ-2-Diabetes erkrankt. Alle bekannten Trends wurden bestätigt. Diabetes betrifft häufiger Männer und Ältere. Bei Frauen nähert sich das Risiko dem der Männer mit zunehmendem Alter immer schneller an. Prävalenz und Inzidenz steigen zwischen dem 15. und 79. Lebensjahr stark an. Die Inzidenz erreicht um das 85. Lebensjahr ihren Höhepunkt.

Die Studie besticht als Totalerhebung eines riesigen Kollektivs mit solider Methodik. Sie ist aber auch ein politisches Fanal. Schaut man sich andere Bereiche an, wo 10\% der Bevölkerung von einem Nachteil betroffen sind, agieren Regierungen sehr schnell.

Prof. Dr. med. P. Schwarz

- Tamayo T, Brinks $R$, Hoyer $A$ et al. The prevalence and incidence of diabetes in Germany. Dtsch Arztebl Int. 2016;113:177-82 